\title{
Estimates of Variability Studies for Various Leaf Yield Attributing Traits in Kale (Brassica oleracea L. var. acephala) Genotypes
}

\author{
Sunnia Gorka*, R.K. Samnotra, Sanjeev Kumar, Sandeep Chopra \\ and M. Iqbal Jeelani Bhat
}

University of Agricultural and Horticultural Sciences, Shimoga-577204, Karnataka, India

*Corresponding author

\begin{abstract}
A B S T R A C T
The present investigation entitled "Estimates of variability studies for various leaf yield

Keywords attributing traits in kale (Brassica oleracea L. var. acephala)" comprising of thirty genotypes was carried out at Vegetable Research Farm, Division of Vegetable Science \& Floriculture, FOA, Chatha, SKUAST-J, Main campus, Chatha to estimate variability for

Kale, Mean 12 traits during the year 2015-16 and 2016-17. The field experiment was laid out in performance, Variability Randomized Block Design with three replications, spaced at $60 \mathrm{~cm} \times 45 \mathrm{~cm}$. The observations were recorded on five randomly selected tagged plants. The analysis of

Article Info

Accepted:

20 January 2018

Available Online:

10 February 2018 variance of pooled data revealed significant differences among the genotypes for all the characters studied. Based on the mean performance genotypes namely SJK-02, SJK-03 SJK-04 and SJK-05 were high yielding with regard to number of leaves per plant, number of pickings per plant, duration of pickings and leaf yield per plant \& SJK-19 was also found to be best performers for mean leaf weight per plant. Genotypes namely SJK-10 was found to be the earliest for days to first leaf picking. Thus, revealing that substantial amount of variability in present for various traits among the kale genotypes studied and therefore can be further exploited in further kale improvement programme.
\end{abstract}

\section{Introduction}

Kale (Brassica oleracea L. var. acephala DC.) is leafy green vegetable belonging to cabbage family and is probably the first brassicas to be cultivated and probable progenitor of cabbage, cauliflower, broccoli. It belongs to family Cruciferaae (Khan et al., 2011) and includes leafy kale (var. acephala), thousand head kale (var. ramosa), scotch kale (var. sabellica), marrow stem kale (var. medullosa), palm kale (var. palmifolia), collards (var. viridis) and Chinese kale (var. alboglabra) (Diederichsen, 2001). At present grown extensively throughout all the parts of the world over an area of 2470.27 thousand hectares with a productivity of 29.05 tonnes/hectare. In Jammu and Kashmir, it is being grown over an area of 2460 hectares out of which in Jammu region it occupies an area of 160 hectares with a productivity of 18.78 tonnes/hectare (Anonymous, 2014).

Kale is a very nutritious vegetable, rich in vitamins and minerals particularly vitamin $\mathrm{C}$, pro-vitamin ( $\beta$-carotene \& lutein) and 
minerals and anti oxidants. Consumption of kale juice is determined to raise the HDL levels and lower the LDL levels, and also improve their artherogenic profiles which measured their likelihood of developing coronary artery disease (Kim et al., 2008). Among all 100 of the world's healthiest foods, kale grabs the first position in terms of lutein content and is the primary luteincontaining food in the USDA's National Nutrient Database. Kale is a potent source of glucosinolates and contains about 15-20 different glucosinolates like compounds (Sikora et al., 2007). Kale is a highly cross pollinated vegetable crop and is grown under all the agro climatic conditions from subtropical, temperate to cold arid zones of the $J \& K$ state. The crop is reported rich in diversity due to complex natural intra and inters specific crosses and geographical barriers. Estimates of variability parameters is present in North West Himalayan region and has not been properly documented and characterized for further use in any plant breeding programme. It is a well documented fact that variation shown by available genetic resources for quantitative and quality traits is important for vegetable breeding programme (Esribano et al., 1998). These studies can provide potential genetic resources by elucidating genetic information and relationships between different populations for crop improvement and facilitating the identification of diverse parents to cross in hybrid combinations in order to maximize the expression of heterosis (Purugganan et al., 2000).

\section{Materials and Methods}

The present investigation entitled "Estimates of variability studies for various leaf yield attributing traits in kale (Brassica oleracea L. var. acephala)" was carried out under subtropical conditions of Jammu at Vegetable Experimental Farm, Division of Vegetable
Science and Floriculture, Faculty of Agriculture, Sher-e-Kashmir University of Agricultural Sciences and Technology, Main Campus, Chatha, Jammu (J\&K). The experimental field of Division of Vegetable Science and Floriculture, SKUAST, Jammu is situated at $32^{\circ} 40^{\prime} \mathrm{N}$ latitude and $74^{\circ} 58^{\prime} \mathrm{E}$ longitude and has an elevation of $332 \mathrm{~m}$ above mean sea level.

The experimental material comprised of 30 diverse genotypes of kale locally collected from all the three region of $\mathrm{J} \& \mathrm{~K}$ including Central Institute of Temperate Horticulture \& SKUAST-K. The details of the genotypes along with their source are given below in Table 1.

The experimental material comprised of thirty genotypes of Kale which after raising healthy seedlings were transplanted in Randomized Block Design with three replications during 2015-2016 and 2016-17 at the experimental farm of Division of Vegetable Science and Floriculture, SKUAST-Jammu.

All the thirty genotypes were grown in a plot size of $3.0 \mathrm{~m} \times 1.80 \mathrm{~m}$ with a spacing of 60 $\mathrm{cm}$ row to row and $45 \mathrm{~cm}$ plant to plant. Observations were recorded on various morphological/yield contributing traits on five randomly selecting tagged plants from each treatment in each replication and there means were worked out for statistical analysis as per method given by Panse and Sukhatme (1967).

\section{Results and Discussion}

The analysis of variance for various characters in kale (Brassica oleracea L. var. acephala) during 2015-16, 2016-17 and in pooled analysis showed significant differences among genotypes used in the present investigation for 12 traits under study (Table 2, 3 and 4). 
Table.1 List of genotypes along with the source of procurement

\begin{tabular}{|c|c|c|}
\hline S. No. & Code no. & Source \\
\hline 1. & SJK-01 & SKUAST-K \\
\hline 2. & SJK-02 & Kupwara \\
\hline 3. & SJK-03 & CITH \\
\hline 4. & SJK-04 & Bandipura \\
\hline 5. & SJK-05 & Farmers Friendly Participatory Research Station, Karlah \\
\hline 6. & SJK-06 & SKUAST-K \\
\hline 7. & SJK-07 & Kupwara \\
\hline 8. & SJK-08 & CITH \\
\hline 9. & SJK-09 & Bandipura \\
\hline 10. & SJK-10 & Farmers Friendly Participatory Research Station, Karlah \\
\hline 11. & SJK-11 & Leh \\
\hline 12. & SJK-12 & Leh \\
\hline 13. & SJK-13 & Kanachak \\
\hline 14. & SJK-14 & Sarora \\
\hline 15. & SJK-15 & Marh \\
\hline 16. & SJK-16 & Karloop \\
\hline 17. & SJK-17 & Chenani \\
\hline 18. & SJK-18 & Assar \\
\hline 19. & SJK-19 & SKUAST-K \\
\hline 20. & SJK-20 & SKUAST-K \\
\hline 21. & SJK-21 & SKUAST-K \\
\hline 22. & SJK-22 & SKUAST-K \\
\hline 23. & SJK-23 & CITH \\
\hline 24. & SJK-24 & CITH \\
\hline 25. & SJK-25 & Drass \\
\hline 26. & SJK-26 & SKUAST-K \\
\hline 27. & SJK-27 & SKUAST-K \\
\hline 28. & SJK-28 & SKUAST-K \\
\hline 29. & SJK-29 & CITH \\
\hline 30. & SJK-30 & Pulwama \\
\hline
\end{tabular}


Table.2 Analysis of variance for various quantitative and quality traits in kale (Brassica oleracea L. var. acephala): 2015-16

\begin{tabular}{|l|c|c|c|}
\hline \multirow{2}{*}{\multicolumn{1}{|c|}{ Traits }} & \multicolumn{2}{c|}{ Mean sum of squares } & \multirow{2}{*}{ F-value } \\
\cline { 2 - 3 } & Genotypes & Error & \\
\hline Days to first leaf picking & 46.54 & 3.26 & $14.24^{*}$ \\
\hline Leaf area index & 1.93 & 0.22 & $8.95^{*}$ \\
\hline Mean leaf weight per plant (g) & 8.85 & 4.19 & $2.05^{*}$ \\
\hline Stem thickness (cm) & 2.75 & 0.16 & $16.73^{*}$ \\
\hline Plant height (cm) & 417.15 & 31.97 & $13.05^{*}$ \\
\hline Number of leaves per plant & 927.42 & 89.57 & $10.35^{*}$ \\
\hline Number of nodes per plant & 43.78 & 4.24 & $10.32^{*}$ \\
\hline Intermodal length (cm) & 1.26 & 0.08 & $15.16^{*}$ \\
\hline Number of pickings per plant & 16.04 & 1.44 & $11.12^{*}$ \\
\hline Duration of picking & 479.81 & 85.79 & $5.59^{*}$ \\
\hline Leaf yield per plant (g) & 191878.30 & 36061.02 & $5.32^{*}$ \\
\hline Leaf yield per hectare (q/ha) & 1289.21 & 2423.85 & $5.32^{*}$ \\
\hline
\end{tabular}

Table.3 Analysis of variance for various quantitative and quality traits in kale (Brassica oleracea L. var. acephala): 2016-17

\begin{tabular}{|l|c|c|c|}
\hline \multirow{2}{*}{ Traits } & \multicolumn{2}{|c|}{ Mean sum of squares } & \multirow{2}{*}{ F-value } \\
\cline { 2 - 4 } & Genotypes & Error & $13.40^{*}$ \\
\hline Days to first leaf picking & 46.59 & 3.48 & $7.39^{*}$ \\
\hline Leaf area index & 1.80 & 0.24 & $1.92^{*}$ \\
\hline Mean leaf weight per plant (g) & 5.94 & 3.10 & $15.98^{*}$ \\
\hline Stem thickness (cm) & 2.66 & 0.17 & $11.20^{*}$ \\
\hline Plant height (cm) & 416.44 & 37.19 & $9.77^{*}$ \\
\hline Number of leaves per plant & 852.49 & 87.25 & $10.99^{*}$ \\
\hline Number of nodes per plant & 41.02 & 3.73 & $19.02^{*}$ \\
\hline Intermodal length (cm) & 1.15 & 0.06 & $10.85^{*}$ \\
\hline Number of pickings per plant & 14.54 & 1.34 & $4.68^{*}$ \\
\hline Duration of picking & 416.11 & 88.89 & $4.38^{*}$ \\
\hline Leaf yield per plant (g) & 193132.09 & 44085.53 & $4.38^{*}$ \\
\hline Leaf yield per hectare (q/ha) & 12981.43 & 2963.19 & \\
\hline
\end{tabular}


Table.4 Pooled analysis of variance for various quantitative and quality traits in kale (Brassica oleracea L. var. acephala)

\begin{tabular}{|c|c|c|c|}
\hline \multirow[t]{2}{*}{ Traits } & \multicolumn{2}{|c|}{ Mean sum of squares } & \multirow[t]{2}{*}{ F-value } \\
\hline & Genotypes & Error & \\
\hline Days to first leaf picking & 46.47 & 3.30 & $14.09 *$ \\
\hline Leaf area index & 1.86 & 0.22 & $8.29 *$ \\
\hline Mean leaf weight per plant (g) & 6.34 & 2.81 & $2.25^{*}$ \\
\hline Stem thickness (cm) & 2.70 & 0.16 & $17.07 *$ \\
\hline Plant height (cm) & 413.70 & 32.09 & $12.89^{*}$ \\
\hline Number of leaves per plant & 884.78 & 88.07 & $10.04^{*}$ \\
\hline Number of nodes per plant & 42.31 & 3.90 & $10.84 *$ \\
\hline Intermodal length (cm) & 1.19 & 0.06 & $19.27^{*}$ \\
\hline Number of pickings per plant & 15.16 & 1.27 & $11.96^{*}$ \\
\hline Duration of picking & $4 * 39.60$ & 80.61 & 5.45 \\
\hline Leaf yield per plant (g) & 184182.80 & 33915.89 & $5.43 *$ \\
\hline Leaf yield per hectare (q/ha) & 12379.86 & 2279.68 & $5.43^{*}$ \\
\hline
\end{tabular}


Table.5 Mean performance of various quantitative and quality traits in kale (Brassica oleracea $\mathrm{L}$ var. acephala) -2015-16

\begin{tabular}{|c|c|c|c|c|c|c|c|c|c|c|c|c|}
\hline $\begin{array}{c}\text { Genotype } \\
\text { (Ideotype of genotype) }\end{array}$ & $\begin{array}{l}\text { Days to } \\
\text { first leaf } \\
\text { picking }\end{array}$ & $\begin{array}{l}\text { Leaf } \\
\text { area } \\
\text { index }\end{array}$ & $\begin{array}{c}\begin{array}{c}\text { Mean leaf } \\
\text { weight/plant } \\
\text { (g) }\end{array}\end{array}$ & $\begin{array}{c}\text { Stem } \\
\text { thickness } \\
(\mathbf{c m})\end{array}$ & $\begin{array}{c}\text { Plant } \\
\text { height } \\
(\mathbf{c m})\end{array}$ & $\begin{array}{l}\text { Number of } \\
\text { leaves/plant }\end{array}$ & $\begin{array}{l}\text { Number of } \\
\text { nodes/plant }\end{array}$ & $\begin{array}{l}\text { Intermodal } \\
\text { length }(\mathbf{c m})\end{array}$ & $\begin{array}{c}\text { Number of } \\
\text { pickings } \\
\text { /plant }\end{array}$ & $\begin{array}{l}\text { Duration of } \\
\text { picking } \\
\text { (days) }\end{array}$ & $\begin{array}{c}\text { Leaf } \\
\text { yield/plant } \\
\text { (g) }\end{array}$ & $\begin{array}{c}\text { Leaf } \\
\text { yield/ha } \\
(q / h a)\end{array}$ \\
\hline SJK-01 (Khanyari -1) & 23.35 & 3.43 & 13.65 & 2.31 & 47.54 & 89.05 & 17.67 & 2.42 & 11.25 & 100.02 & 1302.43 & 337.67 \\
\hline SJK-02 (Khanyari -1) & 24.82 & 2.72 & 14.48 & 2.54 & 39.50 & 102.53 & 10.67 & 3.23 & 10.37 & 94.41 & 1579.59 & 409.52 \\
\hline SJK-03 (Khanyari -1) & 23.28 & 2.79 & 12.33 & 2.41 & 50.80 & 97.86 & 12.00 & 3.87 & 13.32 & 95.48 & 1309.13 & 339.40 \\
\hline SJK-04 (Khanyari -1) & 24.81 & 2.68 & 12.31 & 2.23 & 43.36 & 114.72 & 12.33 & 3.14 & 12.81 & 97.19 & 1509.50 & 391.35 \\
\hline SJK-05 (Khanyari -1) & 25.24 & 3.14 & 12.16 & 3.18 & 68.87 & 123.52 & 17.67 & 3.63 & 11.92 & 99.49 & 1610.09 & 417.43 \\
\hline SJK-06 (G.M.Dari-1) & 24.92 & 3.53 & 13.03 & 2.29 & 38.23 & 80.48 & 16.33 & 2.03 & 7.79 & 80.25 & 1135.64 & 294.43 \\
\hline SJK-07 (G.M.Dari-1) & 22.89 & 4.18 & 12.51 & 3.75 & 54.73 & 74.83 & 19.33 & 2.57 & 7.46 & 70.61 & 1008.78 & 261.53 \\
\hline SJK-08 (G.M.Dari-1) & 23.48 & 4.54 & 14.59 & 2.45 & 38.73 & 86.94 & 18.00 & 1.87 & 7.03 & 73.09 & 1349.28 & 349.81 \\
\hline SJK-09 (G.M.Dari-1) & 22.64 & 4.34 & 14.56 & 4.14 & 28.37 & 88.13 & 8.00 & 2.97 & 6.58 & 73.72 & 1368.87 & 354.89 \\
\hline SJK-10 (G.M.Dari-1) & 21.35 & 4.42 & 13.51 & 2.47 & 54.40 & 75.58 & 14.67 & 3.37 & 6.53 & 75.79 & 1089.78 & 282.53 \\
\hline SJK-11 (Leh local-1) & 21.19 & 4.75 & 15.74 & 2.27 & 23.67 & 91.17 & 9.00 & 2.13 & 9.22 & 103.83 & 1526.18 & 395.68 \\
\hline SJK-12 (Leh local-2) & 28.55 & 2.31 & 9.62 & 2.22 & 39.93 & 95.61 & 16.33 & 2.17 & 9.74 & 87.84 & 1016.98 & 263.66 \\
\hline SJK-13 (Jammu local-1) & 27.74 & 2.46 & 12.43 & 2.32 & 56.63 & 68.86 & 19.33 & 2.67 & 7.55 & 67.43 & 918.42 & 238.11 \\
\hline SJK-14 (Jammu local-2) & 31.53 & 2.48 & 11.03 & 2.60 & 32.90 & 62.68 & 18.67 & 1.50 & 6.97 & 62.62 & 755.24 & 195.80 \\
\hline SJK-15 (Jammu local-3) & 30.88 & 2.90 & 11.47 & 4.55 & 59.67 & 68.27 & 19.00 & 2.87 & 7.43 & 62.89 & 841.41 & 218.14 \\
\hline SJK-16 (Jammu local-4) & 31.97 & 2.28 & 11.30 & 2.41 & 40.97 & 55.77 & 11.67 & 3.09 & 6.53 & 61.82 & 688.24 & 178.43 \\
\hline SJK-17 (Jammu local-5) & 33.67 & 2.47 & 11.69 & 2.10 & 34.83 & 63.72 & 13.00 & 2.30 & 10.17 & 82.78 & 783.37 & 203.09 \\
\hline SJK-18 (Jammu local-6) & 27.62 & 2.14 & 12.24 & 2.09 & 49.33 & 62.60 & 13.00 & 3.40 & 10.80 & 83.18 & 818.35 & 212.16 \\
\hline SJK-19 (Anchari green) & 34.71 & 2.42 & 15.32 & 2.35 & 21.56 & 83.19 & 9.33 & 1.83 & 13.00 & 84.41 & 1346.56 & 349.11 \\
\hline SJK-20 (Kawdari) & 29.26 & 2.36 & 11.56 & 2.10 & 33.73 & 85.82 & 8.33 & 3.47 & 8.83 & 65.89 & 1082.41 & 280.62 \\
\hline SJK-21 (Wantipuri) & 29.80 & 2.26 & 12.12 & 2.65 & 27.91 & 85.80 & 9.67 & 2.44 & 11.13 & 84.33 & 1129.97 & 292.96 \\
\hline SJK-22 (Sag Purple Sel.) & 29.24 & 2.37 & 13.20 & 2.06 & 36.63 & 88.79 & 12.67 & 2.50 & 9.72 & 67.22 & 1254.05 & 325.12 \\
\hline SJK-23 (Japanese Green) & 29.32 & 2.36 & 12.83 & 5.27 & 32.17 & 71.13 & 12.67 & 2.13 & 7.26 & 67.87 & 975.18 & 252.82 \\
\hline SJK-24 (Siberian Kale) & 22.95 & 4.06 & 13.74 & 3.81 & 40.30 & 67.04 & 10.67 & 3.30 & 7.42 & 70.62 & 984.41 & 255.22 \\
\hline SJK-25 (Drass Kale) & 22.35 & 4.02 & 15.96 & 2.40 & 45.60 & 65.39 & 18.67 & 2.17 & 6.75 & 75.28 & 1098.11 & 284.69 \\
\hline SJK-26 (Sag-81) & 22.59 & 3.65 & 15.88 & 2.28 & 46.93 & 59.40 & 14.00 & 2.99 & 6.24 & 88.74 & 999.06 & 259.02 \\
\hline SJK-27 (Sag-88) & 22.20 & 3.21 & 14.97 & 2.49 & 18.09 & 59.61 & 7.33 & 1.76 & 6.20 & 88.31 & 944.53 & 244.88 \\
\hline SJK-28 (Sag-100) & 23.23 & 3.40 & 15.86 & 2.72 & 30.63 & 54.40 & 9.67 & 2.73 & 5.33 & 61.71 & 901.93 & 233.83 \\
\hline SJK-29 (Hanz Hak) & 22.69 & 3.59 & 14.78 & 4.75 & 37.90 & 60.16 & 10.33 & 3.17 & 6.45 & 84.49 & 955.32 & 247.68 \\
\hline SJK-30 (Kashmir local) & 23.16 & 3.36 & 15.18 & 4.99 & 51.20 & 62.50 & 12.00 & 3.83 & 7.03 & 73.64 & 1024.54 & 265.62 \\
\hline General Mean & 26.05 & 3.15 & 13.34 & 2.87 & 40.84 & 78.19 & 13.40 & 2.72 & 8.63 & 79.50 & 1110.24 & 287.84 \\
\hline SE $\mathbf{m} \pm$ & 1.04 & 0.27 & 1.18 & 0.23 & 3.27 & 5.46 & 1.19 & 0.17 & 0.69 & 5.35 & 109.64 & 28.42 \\
\hline CV (\%) & 6.94 & 14.71 & 15.35 & 14.12 & 13.85 & 12.10 & 15.37 & 10.63 & 13.92 & 11.65 & 17.10 & 17.10 \\
\hline CD (at 0.05) & 2.96 & 0.76 & 3.35 & 0.66 & 9.27 & 15.51 & 3.37 & 0.47 & 1.97 & 15.18 & 311.17 & 80.67 \\
\hline
\end{tabular}


Table.6 Mean performance of various quantitative and quality traits in kale (Brassica oleracea L var. acephala) -2016-17

\begin{tabular}{|c|c|c|c|c|c|c|c|c|c|c|c|c|}
\hline $\begin{array}{c}\text { Genotype } \\
\text { (Ideotype of genotype) }\end{array}$ & $\begin{array}{c}\text { Days to } \\
\text { first } \\
\text { leaf } \\
\text { picking }\end{array}$ & $\begin{array}{l}\text { Leaf area } \\
\text { index }\end{array}$ & $\begin{array}{c}\text { Mean leaf } \\
\text { weight/plant } \\
\text { (g) }\end{array}$ & $\begin{array}{c}\text { Stem } \\
\text { thickness } \\
(\mathrm{cm})\end{array}$ & $\begin{array}{c}\text { Plant } \\
\text { height } \\
(\mathrm{cm})\end{array}$ & $\begin{array}{l}\text { Number of } \\
\text { leaves/plant }\end{array}$ & $\begin{array}{l}\text { Number of } \\
\text { nodes/plant }\end{array}$ & $\begin{array}{l}\text { Intermodal } \\
\text { length }(\mathbf{c m})\end{array}$ & $\begin{array}{l}\text { Number of } \\
\text { pickings } \\
\text { /plant }\end{array}$ & $\begin{array}{c}\text { Duration } \\
\text { of } \\
\text { picking } \\
\text { (days) }\end{array}$ & $\begin{array}{c}\text { Leaf } \\
\text { yield/plant } \\
\text { (g) }\end{array}$ & $\begin{array}{c}\text { Leaf } \\
\text { yield/ha } \\
\text { (q/ha) }\end{array}$ \\
\hline SJK-01 (Khanyari -1) & 24.26 & 3.68 & 10.37 & 2.35 & 43.31 & 89.15 & 19.31 & 2.24 & 11.83 & 102.86 & 1007.85 & 261.29 \\
\hline SJK-02 (Khanyari -1) & 25.74 & 2.97 & 13.82 & 2.82 & 42.57 & 109.63 & 12.17 & 3.49 & 10.97 & 97.98 & 1621.50 & 420.39 \\
\hline SJK-03 (Khanyari -1) & 23.87 & 3.04 & 13.23 & 2.62 & 47.46 & 104.96 & 13.35 & 3.55 & 13.02 & 102.38 & 1497.45 & 388.23 \\
\hline SJK-04 (Khanyari -1) & 25.39 & 2.93 & 12.44 & 2.11 & 45.11 & 117.49 & 13.67 & 3.23 & 13.51 & 104.09 & 1565.44 & 405.85 \\
\hline SJK-05 (Khanyari -1) & 25.82 & 3.39 & 11.69 & 3.32 & 68.84 & 126.95 & 18.99 & 3.62 & 12.62 & 104.06 & 1577.91 & 409.09 \\
\hline SJK-06 (G.M.Dari-1) & 26.17 & 3.78 & 13.89 & 2.56 & 33.34 & 87.58 & 17.58 & 1.89 & 8.49 & 87.15 & 1301.39 & 337.40 \\
\hline SJK-07 (G.M.Dari-1) & 24.80 & 4.43 & 13.70 & 4.06 & 53.33 & 81.93 & 20.32 & 2.62 & 8.16 & 76.51 & 1201.34 & 311.46 \\
\hline SJK-08 (G.M.Dari-1) & 24.73 & 4.71 & 14.44 & 2.63 & 40.49 & 94.04 & 19.65 & 2.06 & 7.77 & 79.99 & 1453.44 & 376.82 \\
\hline SJK-09 (G.M.Dari-1) & 23.23 & 4.43 & 14.59 & 4.39 & 29.30 & 95.23 & 9.36 & 3.02 & 7.04 & 80.62 & 1479.31 & 383.52 \\
\hline SJK-10 (G.M.Dari-1) & 22.27 & 4.67 & 13.88 & 2.68 & 54.46 & 83.34 & 15.23 & 3.56 & 7.03 & 82.69 & 1235.41 & 320.29 \\
\hline SJK-11 (Leh local-1) & 22.44 & 4.91 & 14.76 & 2.48 & 24.40 & 97.94 & 10.20 & 2.32 & 10.05 & 99.40 & 1536.77 & 398.42 \\
\hline SJK-12 (Leh local-2) & 29.80 & 2.56 & 11.46 & 2.43 & 41.05 & 102.04 & 17.61 & 2.26 & 10.34 & 94.74 & 1274.67 & 330.47 \\
\hline SJK-13 (Jammu local-1) & 28.32 & 2.71 & 11.64 & 2.53 & 57.90 & 76.96 & 20.26 & 2.86 & 8.28 & 74.33 & 968.18 & 251.01 \\
\hline SJK-14 (Jammu local-2) & 32.78 & 2.73 & 12.15 & 2.81 & 33.26 & 64.78 & 19.54 & 1.69 & 7.41 & 69.52 & 854.27 & 221.48 \\
\hline SJK-15 (Jammu local-3) & 32.03 & 3.15 & 11.24 & 4.76 & $\mathbf{5 8 . 9 8}$ & 75.37 & 19.27 & 3.06 & 8.13 & 68.12 & 921.04 & 238.79 \\
\hline SJK-16 (Jammu local-4) & 33.22 & 2.59 & 11.93 & 2.62 & 43.77 & 62.87 & 13.23 & 3.28 & 7.17 & 65.39 & 815.46 & 211.41 \\
\hline SJK-17 (Jammu local-5) & 34.59 & 3.05 & 10.65 & 2.31 & 35.21 & 71.22 & 14.17 & 2.47 & 10.87 & 91.68 & 819.52 & 212.47 \\
\hline SJK-18 (Jammu local-6) & 28.87 & 2.39 & 11.99 & 2.30 & 52.69 & 69.70 & 14.64 & 3.59 & 11.50 & 88.41 & 896.11 & 232.32 \\
\hline SJK-19 (Anchari green) & 34.96 & 2.67 & 15.65 & 2.56 & 22.13 & 90.29 & 10.91 & 2.02 & 12.00 & 78.97 & 1504.32 & 390.01 \\
\hline SJK-20 (Kawdari) & 30.18 & 2.71 & 12.45 & 2.31 & 35.86 & 92.92 & 9.84 & 3.56 & 9.53 & 72.79 & 1253.57 & 325.00 \\
\hline SJK-21 (Wantipuri) & 30.72 & 2.51 & 13.00 & 2.86 & 26.70 & 92.90 & 10.40 & 2.49 & 11.83 & 91.23 & 1304.50 & 338.20 \\
\hline SJK-22 (Sag Purple Sel.) & 30.16 & 2.55 & 13.56 & 2.27 & 36.17 & 94.89 & 13.84 & 2.59 & 10.42 & 74.12 & 1373.33 & 356.05 \\
\hline SJK-23 (Japanese Green) & 29.90 & 2.54 & 13.12 & 5.15 & 32.80 & 77.23 & 14.04 & 2.32 & 8.02 & 74.77 & 1077.58 & 279.37 \\
\hline SJK-24 (Siberian Kale) & 24.20 & 4.31 & 13.06 & 4.02 & 43.36 & 74.14 & 12.38 & 3.49 & 7.46 & 78.52 & 1039.97 & 269.62 \\
\hline SJK-25 (Drass Kale) & 23.26 & 4.27 & 13.36 & 2.61 & 45.44 & 71.49 & 19.25 & 2.36 & 7.35 & 80.51 & 1026.26 & 266.07 \\
\hline SJK-26 (Sag-81) & 23.17 & 4.04 & 14.28 & 2.49 & 48.58 & 66.50 & 15.25 & 3.18 & 6.27 & 88.98 & 1023.72 & 265.41 \\
\hline SJK-27 (Sag-88) & 23.11 & 3.46 & 14.52 & 2.67 & 16.72 & 66.04 & 8.48 & 1.95 & 7.57 & 95.21 & 1020.55 & 264.59 \\
\hline SJK-28 (Sag-100) & 24.15 & 3.55 & 14.87 & 2.93 & 30.07 & 60.50 & 10.88 & 2.72 & 5.81 & 68.61 & 947.85 & 245.74 \\
\hline SJK-29 (Hanz Hak) & 23.61 & 3.77 & 13.84 & 4.96 & 39.20 & 67.26 & 11.74 & 3.32 & 7.15 & 91.39 & 1008.55 & 261.48 \\
\hline SJK-30 (Kashmir local) & 23.19 & 3.50 & 15.69 & 5.13 & 48.77 & 79.27 & 13.22 & 3.67 & 7.73 & 77.20 & 1291.05 & 334.72 \\
\hline General Mean & 26.96 & 3.40 & 13.18 & 3.06 & 41.04 & 84.82 & 14.63 & 2.82 & 9.18 & 84.74 & 1196.61 & 310.23 \\
\hline SE $\mathbf{m} \pm$ & 1.08 & 0.29 & 1.02 & 0.24 & 3.52 & 5.39 & 1.11 & 0.14 & 0.67 & 5.44 & 121.22 & 31.43 \\
\hline CV (\%) & 6.92 & 14.51 & 13.36 & 13.35 & 14.86 & 11.01 & 13.21 & 8.74 & 12.61 & 11.13 & 17.55 & 17.55 \\
\hline CD (at 0.05) & 3.06 & 0.89 & 2.88 & 0.67 & 9.99 & 15.31 & 3.17 & 0.40 & 1.90 & 15.45 & 344.05 & 89.20 \\
\hline
\end{tabular}


Table.7 Pooled mean performance of various quantitative and quality traits in kale (Brassica oleracea L var. acephala)

\begin{tabular}{|c|c|c|c|c|c|c|c|c|c|c|c|c|}
\hline $\begin{array}{c}\text { Genotype } \\
\text { (Ideotype of genotype) }\end{array}$ & $\begin{array}{l}\text { Days to } \\
\text { first } \\
\text { leaf } \\
\text { picking }\end{array}$ & $\begin{array}{l}\text { Leaf area } \\
\text { index }\end{array}$ & $\begin{array}{c}\text { Mean leaf } \\
\text { weight/plant } \\
\text { (g) }\end{array}$ & $\begin{array}{c}\text { Stem } \\
\text { thickness } \\
(\mathbf{c m})\end{array}$ & $\begin{array}{c}\text { Plant } \\
\text { height } \\
(\mathrm{cm})\end{array}$ & $\begin{array}{l}\text { Number of } \\
\text { leaves/plant }\end{array}$ & $\begin{array}{l}\text { Number of } \\
\text { nodes/plant }\end{array}$ & $\begin{array}{l}\text { Intermodal } \\
\text { length }(\mathrm{cm})\end{array}$ & $\begin{array}{l}\text { Number of } \\
\text { pickings } \\
\text { /plant }\end{array}$ & $\begin{array}{c}\text { Duration } \\
\text { of } \\
\text { picking } \\
\text { (days) }\end{array}$ & $\begin{array}{c}\text { Leaf } \\
\text { yield/plant } \\
(\mathrm{g})\end{array}$ & $\begin{array}{c}\text { Leaf } \\
\text { yield/ha } \\
\text { (q/ha) }\end{array}$ \\
\hline SJK-01 (Khanyari -1) & 23.81 & 3.56 & 12.01 & 2.33 & 45.42 & 89.10 & 18.49 & 2.34 & 11.54 & 101.44 & 1155.14 & 299.48 \\
\hline SJK-02 (Khanyari -1) & 25.29 & 2.85 & 14.15 & 2.68 & 41.04 & 106.08 & 11.42 & 3.37 & 10.67 & 96.19 & 1600.54 & 414.96 \\
\hline SJK-03 (Khanyari -1) & 23.58 & 2.92 & 12.78 & 2.52 & 49.13 & 101.41 & 12.68 & 3.71 & 13.17 & 98.93 & 1403.29 & 363.82 \\
\hline SJK-04 (Khanyari -1) & 25.11 & 2.81 & 12.37 & 2.18 & 44.24 & 116.10 & 13.01 & 3.19 & 13.16 & 100.64 & 1537.47 & 398.60 \\
\hline SJK-05 (Khanyari -1) & 25.54 & 3.27 & 11.92 & 3.25 & 68.85 & 125.23 & 18.33 & 3.63 & 12.27 & 101.78 & 1594.00 & 413.26 \\
\hline SJK-06 (G.M.Dari-1) & 25.55 & 3.66 & 13.46 & 2.43 & 35.79 & 84.03 & 16.96 & 1.97 & 8.14 & 83.70 & 1218.51 & 315.91 \\
\hline SJK-07 (G.M.Dari-1) & 23.85 & 4.31 & 13.11 & 3.91 & 54.04 & 78.38 & 19.83 & 2.60 & 7.81 & 73.73 & 1105.06 & 286.50 \\
\hline SJK-08 (G.M.Dari-1) & 24.11 & 4.63 & 14.52 & 2.54 & 39.61 & 90.49 & 18.83 & 1.97 & $\mathbf{7 . 4 0}$ & 76.54 & 1401.36 & 363.31 \\
\hline SJK-09 (G.M.Dari-1) & 22.94 & 4.39 & 14.57 & 4.27 & 28.84 & 91.68 & 8.69 & 3.00 & 6.81 & 77.17 & 1424.09 & 369.21 \\
\hline SJK-10 (G.M.Dari-1) & 21.81 & 4.55 & 13.70 & 2.58 & 54.44 & 79.46 & 14.95 & 3.47 & 6.78 & 79.24 & 1162.59 & 301.41 \\
\hline SJK-11 (Leh local-1) & 21.82 & 4.84 & 15.25 & 2.38 & 24.03 & 94.55 & 9.60 & 2.23 & 9.63 & 101.62 & 1531.47 & 397.05 \\
\hline SJK-12 (Leh local-2) & 29.18 & 2.44 & 10.54 & 2.33 & 40.49 & 98.83 & 16.98 & 2.22 & 10.04 & 91.29 & 1145.83 & 297.07 \\
\hline SJK-13 (Jammu local-1) & 28.04 & 2.59 & 12.03 & 2.43 & 57.27 & 72.91 & 19.80 & 2.77 & 7.91 & 70.88 & 943.30 & 244.56 \\
\hline SJK-14 (Jammu local-2) & 32.16 & 2.61 & 11.59 & 2.71 & 33.08 & 63.73 & 19.11 & 1.60 & 7.19 & 66.07 & 804.75 & 208.64 \\
\hline SJK-15 (Jammu local-3) & 31.46 & 3.03 & 11.35 & 4.66 & 59.32 & 71.82 & 19.14 & 2.97 & 7.78 & 65.50 & 881.22 & 228.47 \\
\hline SJK-16 (Jammu local-4) & 32.60 & 2.44 & 11.62 & 2.52 & 42.37 & 59.32 & 12.45 & 3.19 & 6.85 & 63.61 & 751.84 & 194.92 \\
\hline SJK-17 (Jammu local-5) & 34.14 & 2.76 & 11.17 & 2.21 & 35.02 & 67.47 & 13.59 & 2.39 & 10.52 & 87.23 & 801.44 & 207.78 \\
\hline SJK-18 (Jammu local-6) & 28.25 & 2.27 & 12.11 & 2.20 & 51.01 & 66.15 & 13.83 & 3.50 & 11.15 & 85.80 & 857.23 & 222.24 \\
\hline SJK-19 (Anchari green) & 34.84 & 2.55 & 15.49 & 2.46 & 21.84 & 86.74 & 10.13 & 1.93 & 12.50 & 81.69 & 1425.44 & 369.56 \\
\hline SJK-20 (Kawdari) & 29.73 & 2.54 & 12.00 & 2.21 & 34.79 & 89.37 & 9.09 & 3.52 & 9.18 & 69.34 & 1167.99 & 302.81 \\
\hline SJK-21 (Wantipuri) & 30.26 & 2.39 & 12.57 & 2.76 & 27.30 & 89.35 & 10.04 & 2.47 & 11.48 & 87.78 & 1217.23 & 315.58 \\
\hline SJK-22 (Sag Purple Sel.) & 29.70 & 2.47 & 13.38 & 2.17 & 36.40 & 91.84 & 13.26 & 2.55 & 10.07 & 70.67 & 1313.69 & 340.59 \\
\hline SJK-23 (Japanese Green) & 29.61 & 2.46 & 12.97 & 5.22 & 32.48 & 74.18 & 13.36 & 2.23 & 7.64 & 71.32 & 1026.38 & 266.10 \\
\hline SJK-24 (Siberian Kale) & 23.58 & 4.19 & 13.40 & 3.92 & 41.83 & 70.59 & 11.53 & 3.40 & 7.44 & 74.57 & 1012.18 & 262.42 \\
\hline SJK-25 (Drass Kale) & 22.81 & 4.15 & 14.66 & 2.51 & 45.52 & 68.44 & 18.96 & 2.27 & 7.05 & 77.90 & 1062.18 & 275.38 \\
\hline SJK-26 (Sag-81) & 22.88 & 3.85 & 15.08 & 2.39 & 47.75 & 62.95 & 14.63 & 3.09 & 6.26 & 88.86 & 1011.39 & 262.21 \\
\hline SJK-27 (Sag-88) & 22.66 & 3.34 & 14.75 & 2.58 & 17.40 & 62.82 & 7.91 & 1.86 & 6.89 & 91.76 & 982.54 & 254.73 \\
\hline SJK-28 (Sag-100) & 23.70 & 3.48 & 15.37 & 2.83 & 30.35 & $\mathbf{5 7 . 4 5}$ & 10.28 & 2.73 & 5.57 & 65.16 & 924.89 & 239.79 \\
\hline SJK-29 (Hanz Hak) & 23.16 & 3.68 & 14.31 & 4.86 & 38.55 & 63.71 & 11.04 & 3.25 & 6.80 & 87.94 & 981.94 & 254.58 \\
\hline SJK-30 (Kashmir local) & 23.18 & 3.43 & 15.43 & 5.06 & 49.98 & 70.88 & 12.62 & 3.76 & 7.38 & $\mathbf{7 5 . 4 2}$ & 1157.80 & 300.17 \\
\hline General Mean & 26.51 & 3.28 & 13.26 & 2.97 & 40.94 & 81.50 & 14.02 & 2.77 & 8.90 & 82.13 & 1153.43 & 299.04 \\
\hline SE $\mathbf{m} \pm$ & 1.05 & 0.27 & 0.97 & 0.23 & 3.27 & 5.42 & 1.14 & 0.14 & 0.65 & 5.18 & 106.33 & 27.56 \\
\hline CV (\%) & 6.85 & 14.72 & 12.65 & 13.39 & 13.84 & 11.52 & 14.10 & 8.98 & 12.65 & 10.93 & 15.96 & 15.97 \\
\hline CD (at 0.05) & 2.98 & 0.78 & 2.75 & 0.65 & 9.28 & 15.38 & 3.24 & 0.41 & 1.85 & 14.71 & 301.77 & 78.24 \\
\hline
\end{tabular}


These differences indicated the presence of substantial amount of variability found for these traits and considerable scope for improvement of various characters namely days to first leaf picking, leaf area index, mean leaf weight per plant, stem thickness, plant height, number of leaves per plant, number of nodes per plant, internodal length, number of pickings per plant and leaf yield per plant in the material under study. These results are in accordance with the findings of earlier researcher's viz. Ali et al., (2017), Tripathi et al., (2015), Synrem et al., (2014) and Akbar et al., (2003), who also observed wide range of variability for various traits in Brassica species.

Based on the mean performance of genotypes with respect to various leaf and seed yield attributing traits, during 2015-16 (Table 5), genotypes namely SJK-02, SJK-03, SJK-04, SJK-08, SJK09, SJK-11, SJK-19 gave leaf yield statistically at par with SJK-05 which was the best performer. SJK-05 also found to be one of the best performers for plant height and number of leaves per plant; during 201617 (Table 6), genotype namely SJK-03, SJK04, SJK-05, SJK-08, SJK-09, SJK-11 and SJK-19 gave leaf yield statistically at par with SJK-02 which was the best performer; in pooled analysis (Table 7) genotypes namely SJK-03, SJK-04, SJK-05, SJK08, SJK-09, SJK-11, SJK-19 and SJK-22 gave leaf yield statistically at par with SJK-02 which was the best performer. SJK-05 was found to be one of the best performers for various leaf yield attributing traits including plant height, number of leaves per plant and duration of picking indicating that these genotypes can be further exploited for further use in breeding programme.

Considerable amount of variability was observed with respect to range and pronounced mean values for plant height, number of leaves per plant, duration of picking and leaf yield per plant indicated the scope of selection of these traits. Similar findings were obtained by other workers namely Saleem et al., (2017), Khan et al., (2010) and Malode et al., (2010) for number of leaves per plant, plant height, number of nodes per plant and duration of picking. The results obtained in the present investigation indicated that considerable variability and diversity was available for converging the elite allelic resources present in the genotypes through planned breeding and selection approaches to recover high yielding segregants that would possess good quality characteristics as well. Improvement over existing varieties is a continuous process in plant breeding.

\section{References}

Akbar, M. T., Mahmood, M., Yaqub, M., Anwar, M. A. and Iqbal, N. 2003. Variability, correlation and path coefficient studies in summer mustard (Brassica juncea L.). Asian Journal of Plant Sciences, 2(9): 696698.

Anonymous 2014. Area and production of crop in J\&K. Annual report, Department of Agriculture, Jammu. pp. 89.

Diederichsen, A. 2001. Cruciferae: Brassica. In: Hanelt P, Institute of Plant Genetics and Crop Plant Research (eds) Mansfeld's Encyclopedia of agricultural and horticultural crops. Springer, Berlin, pp. 1435-1446.

Escribano, M. R., Santalla, M., Casquero, P. A. and Ron, A. D. E. 1998. Patterns of genetic diversity in landraces of common bean (Phaseolus vulgaris L.) from Galicia. Plant Breeding, 117: 49-56.

Khan, S. H., Ahmed, N., Jabeen, N., Chatoo, M. A. And Hussain, K. 2010. Biodiversity of kale (Brassica oleracea var. acephala) in Kashmir valley. The Asian Journal of Horticulture, 5(1): 208-210.

Khan, W. M., Munir, I., Farhatullah, Arif, M., Iqbal, A., Ali, I., Ahmad, D., Ahmad, M., Mian, A., Bakht, J., Inamullah \& Swati, Z. A. 2011. Assessment of genetic diversity of 
Brassica juncea germplasm using Randomly Amplified Polymorphic DNA (RAPD) markers. African Journal of Biotechnology, 10: 3654-3658.

Kim, S. Y., Yoon, S., Kwon, S. M., Park, K. S. and Lee-Kim, Y. C. 2008. Kale juice improves coronary artery disease risk factors in hypercholesterolemic men. Biomedical and Environmental Sciences, 21(2): 91-97.

Malode, S. N. and Shelke, P. 2010. Morphological, phenological and anatomical studies in yellow seeded mutant Brassica juncea, Bionano Frontier, 3(2): 172-177.

Panse, V. G. And Sukhatmea, P. V. (eds.). 1967. Statistical Methods for Agricultural Workers, pp.361. ICAR, New Delhi, India.

Purugganan, M. D., Boyles, A. L. and Suddith, J. I. 2000. Variation and selection at the cauliflower floral homeotic gene accompanying the evolution of domesticated Brassica oleracea. Genetics, 155(2): 855-862.

Saleem, V. R., Jan, N., Atif, S. A., Khurshid, M. J., Khan, H., Abdullah, S. A., Jahanzaib, M., Ahmed, M., Ullah, S. F., Iqbal, A.,
Naqi, S., Ilyas, M., Ali, N. and Rabbani, M. A. 2017. Multivariate based variability within diverse Indian mustard (Brassica juncea L.) genotypes. Open Journal of Genetics, 7: 69-83.

Sikora, E., Cieslik, E., Leszczynska, T., FilipiakFlorkiewicz, A., Pisulewski, P. M. 2007. The antioxidant activity of selected cruciferous vegetables subjected to aquathermal processing. Food Chemistry, 107(1):55-59.

Synrem, G. J., Rangare, N. R., Myrthong, I. And Bahadure, D. M. 2014. Variability studies in intra specific crosses of Indian mustard (Brassica juncea L.) [Czen and Coss.] genotypes. International Journal of Agriculture and Veterinary Science, 7: 2932.

Tripathi,N., Kumar, K. and Verma, O. P. 2015. Genetic Variability, Heritability and Genetic Advance in Indian mustard (Brassica juncea L. Czern and Coss.) for Seed Yield and it's Contributing Attributes Under Normal and Saline/Alkaline Condition. International Journal of Science and Research, 4(8): 983-985.

\section{How to cite this article:}

Sunnia Gorka, R.K. Samnotra, Sanjeev Kumar, Sandeep Chopra and Iqbal Jeelani Bhat, M. 2018. Estimates of Variability Studies for Various Leaf Yield Attributing Traits in Kale (Brassica oleracea L. var. acephala) Genotypes. Int.J.Curr.Microbiol.App.Sci. 7(02): 2145-2154.

doi: https://doi.org/10.20546/ijcmas.2018.702.257 